\title{
Endoleak accidentally discovered in congestive heart failure patient
}

\author{
Serhat Akay $\cdot$ Huriye Akay $\cdot$ Gulsum Limon
}

Received: 10 February 2010/ Accepted: 24 February 2010/Published online: 19 March 2010 (C) SIMI 2010

\begin{abstract}
A 62-year-old woman with a history of congestive heart failure, chronic obstructive pulmonary disease and endovascular stenting for ascending arch and descending aortic aneurysm was admitted to the university emergency department (ED) with a 1 day history of increased dyspnea. On physical examination, she had bronchospasm diffusely in both lungs with rales in the lower segments. She was started on intravenous nitrates, and furosemide along with bronchodilators that relieved the symptoms. On an upright posteroanterior chest X-ray study, mediastinal enlargement with signs of congestive heart failure along with an endovascular stent starting from the root of the aorta down to the diaphragmatic portion of the aorta were noted (Fig. 1). In order to rule out possible expansion of the aortic aneurysm, a computed tomography (CT scan) of the thorax and the abdomen with intravenous contrast was performed. On the CT scan, an aneurysmal sac around the graft with leaking of the contrast medium at the distal end of stent was noted (Fig. 2). She was diagnosed with type 1
\end{abstract}

\section{S. Akay}

Department of Emergency Medicine, Izmir Bozyaka Training and Research Hospital, Izmir, Turkey

\section{H. Akay}

Department of Emergency Medicine, Ankara Diskapi Yildirim Beyazit Research and Training Hospital, Ankara, Turkey

\section{G. Limon}

Department of Emergency Medicine, Dokuz Eylul University Hospital, Izmir, Turkey

\section{S. Akay (ه)}

1738 sk no:21/1 d:9 Karsiyaka, Izmir, Turkey

e-mail: howls_Castle@mynet.com endoleak, and vascular surgery was consulted. They recommended out-patient treatment, and the patient was discharged home.

Endovascular aortic repair (EVAR) was introduced in the early 1990s by Parodi et al., and it's becoming a more regular treatment for aneurysms. Endoleaks are defined as blood flow external to the stent-graft and inside the aneurysm, and they occur in one-fourth of patients [1]. They usually do not cause symptoms as they are detected on routine checkups or accidentally discovered. Five types of endoleaks are defined, with each having a different treatment approach [2]. In type 1A endoleak, blood flow is seen in the proximal stent while in type $1 \mathrm{~B}$ endoleak, it is seen from the distal stent. Distal site endoleaks do not have

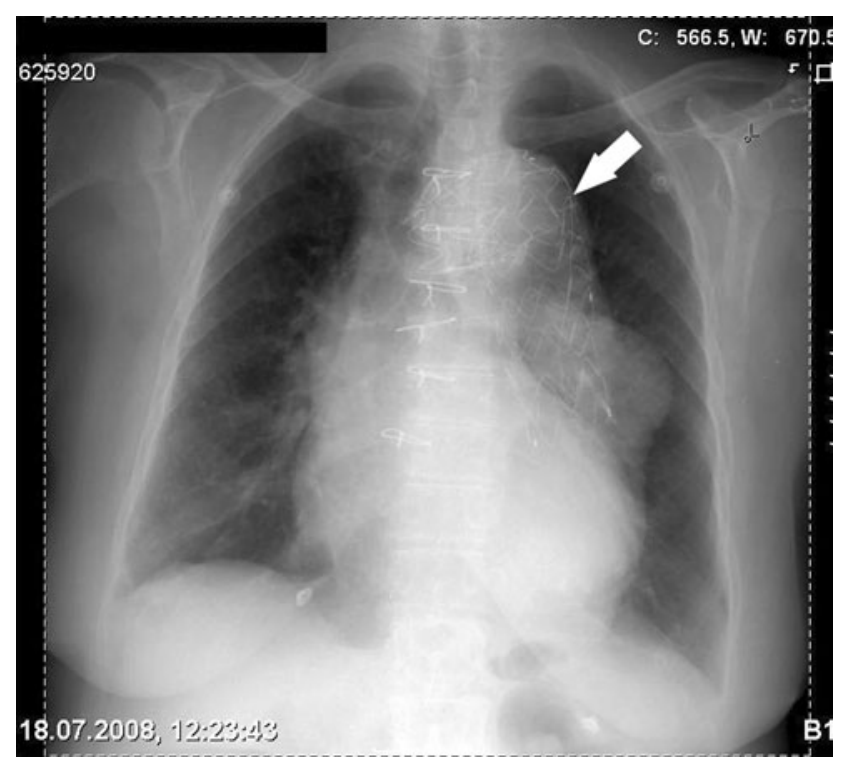

Fig. 1 Pulmonary congestion and EVAT seen on antero posterior chest X-ray 


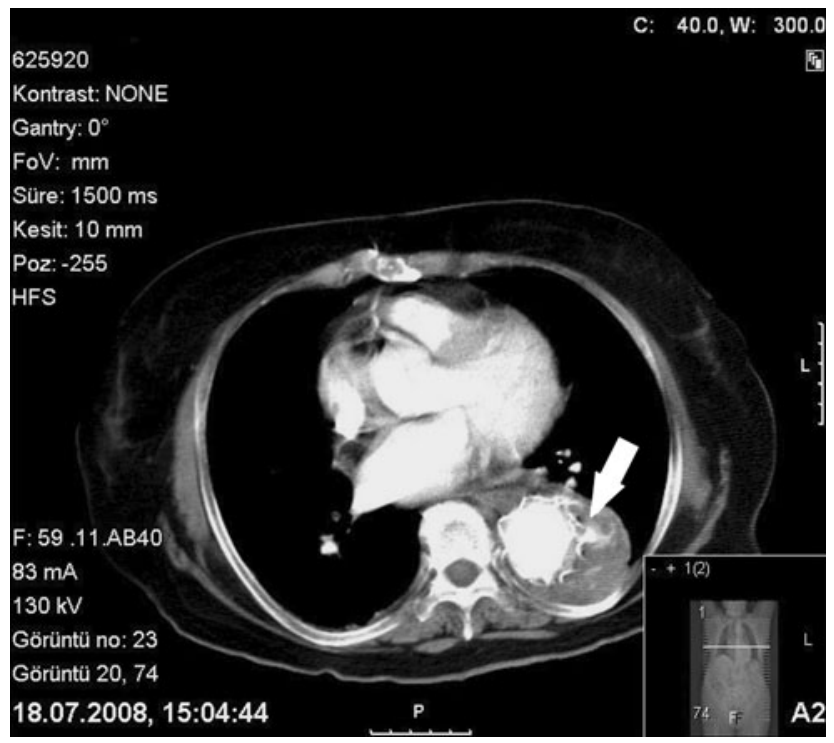

Fig. 2 Type 1B endoleak on thoracoabdominal computed tomography

significant risk of rupture compared to proximal site leaks. Type 2 endoleaks correspond to retrograde filling of the aneurysm sac from lumbar arteries or the inferior mesenteric artery. Type 3 endoleaks consist of hemorrhage from the aorta to the aneurysm due to separation of components of the stent. Type 4 endoleaks are caused by the porosity of the graft, and leakage from the graft. Type 5 leaks are defined as a continued aneurysm expansion without any obvious endoleak on CT scan or angiography. Rupture is observed more often in type 1 and 3 endoleaks [3]. In the EUROSTAR study, $42 \%$ of endoleaks were resolved within 6 months [4].

Diagnosis of endoleaks are often made by CT angiogram on routine checkups. Persisting endoleaks have different treatment approaches dependent on the type of endoleak. Emergency physicians may detect endoleak in patients with EVAR, and will best determine appropriate treatment in consultation with the cardiovascular surgeon, and depending upon the type of the endoleak.

Conflict of interest None.

\section{References}

1. Stavropoulos SW, Charagundla SR (2007) Imaging techniques for detection and management of endoleaks after endovascular aortic aneurysm repair. Radiology 243:641-655

2. Golzarian J, Maes EB, Sun S (2005) Endoleak: treatment options. Tech Vasc Interv Radiol 8:41-49

3. Choke E, Thompson M (2004) Endoleak after endovascular aneurysm repair: current concepts. J Cardiovasc Surg (Torino) 45:349-366

4. Cuypers P, Buth J, Harris PL, Gevers E, Lahey R (1999) Realistic expectations for patients with stent-graft treatment of abdominal aortic aneurysms. Results of a European multicentre registry. Eur J Vasc Endovasc Surg 17:507-516 